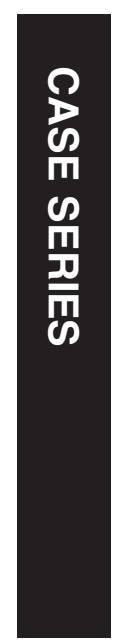

\section{Optical coherence tomography angiography in paracentral acute middle maculopathy secondary to central retinal vein occlusion}

\begin{abstract}
Purpose To report the clinical course and the optical coherence tomography angiography (OCTA) findings of patients presenting with paracentral acute middle maculopathy (PAMM) and central retinal vein occlusion (CRVO). Methods Retrospective case series. Clincal records and multimodal imaging findings of patients presenting with PAMM and CRVO were reviewed.
\end{abstract}

Results Three eyes of three patients ( 2 males; mean age: 66 years) were included in the study. Mean follow-up was 9 months and images using OCTA (AngioVue OCT angiography system, Optovue, Inc., Fremont, CA, USA) were available at the last follow-up visit. During follow-up, best corrected visual acuity (BCVA) of case 1 was unchanged at 85 ETDRS letters and OCTA revealed a mild attenuation of the perifoveal deep capillary plexus (DCP); in case 2, BCVA changed from 83 to 77 ETDRS letters and OCTA revealed patchy areas of attenuation and pruning of the DCP; in case 3, BCVA decreased from 26 to 8 ETDRS letters and OCTA revealed extensive areas of DCP dropout.

Conclusion The natural course of visual acuity in patients with PAMM secondary to CRVO may vary. In these patients, the extent of DCP dropout on OCTA may reflect the extent of visual acuity impairment.

Eye (2016) 30, 888-893; doi:10.1038/eye.2016.57; published online 1 April 2016

\section{Introduction}

With the advent of the spectral domain optical coherence tomography (SD-OCT) technology,
G Casalino ${ }^{1,2}$, M Williams ${ }^{1}$, C McAvoy ${ }^{1}$, F Bandello ${ }^{2}$ and $U$ Chakravarthy ${ }^{1}$ the appearance of abnormal bands of hyperreflectivity have been identified as markers of retinal ischemia. ${ }^{1}$ Paracentral acute middle maculopathy (PAMM) is a recently described manifestation presenting with hyperreflective band-like lesions within the midretina on SD-OCT. ${ }^{2}$ Although it was initially described by Sarraf et $a l^{2}$ as a variant of acute macular neuroretinopathy, PAMM is currently considered a clinical finding related to ischemia of the deep retinal vascular layers. ${ }^{3}$ This finding has been reported to be either idiopathic occurring as an isolated event in otherwise healthy subjects ${ }^{2}$ or secondary to a large spectrum of retinal vascular disorders and systemic conditions including central retinal vein occlusion (CRVO). ${ }^{4,5}$

In this report, we describe the clinical course and imaging findings that include the use of OCT angiography (OCTA; AngioVue OCT angiography system, Optovue, Inc.) of three patients with PAMM secondary to CRVO.

\section{Case reports}

Case 1

A 55-year-old woman was referred for sudden onset paracentral scotoma in the right eye (RE) (Figure 1). She presented with hypercholesterolemia and a history of transient ischemic attack. Best corrected visual acuity (BCVA) was 85 ETDRS letters in both eyes. Fundus examination of the RE revealed a CRVO. No abnormalities were detected in the fellow eye. On SD-OCT, the RE showed typical features of PAMM. After 16 months of follow-up, BCVA was unchanged in both eyes; signs of the 

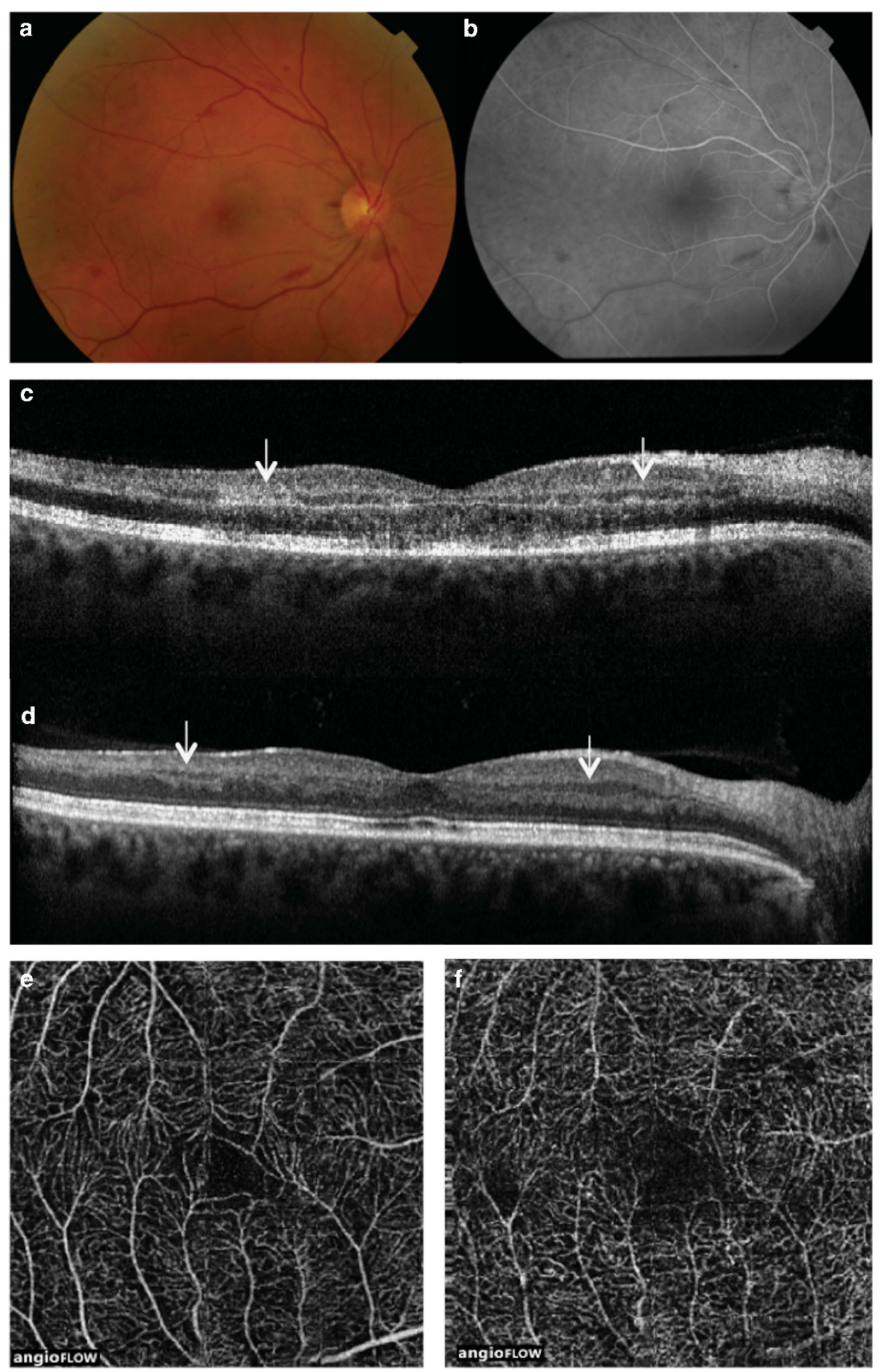

Figure 1 Case 1. Multimodal imaging of right eye. At presentation, BCVA was 85 ETDRS letters; color fundus photograph (CFP) (a) revealed venous engorgement and widespread retinal hemorrhages; fluorescein angiography (FA) (b) showed delayed venous filling; SD-OCT scan (c) showed hyperreflective band-like lesions involving the middle layers of the retina at the level of the INL (arrows). After 16 months, BCVA was stable at 85 ETDRS letters; SD-OCT scan (d) revealed thinning of the INL (arrows); OCTA showed no abnormalities in the SCP (e) and a mild attenuation of the perifoveal DCP (f). 
CRVO were resolved but the patient's scotoma remained unchanged. SD-OCT revealed thinning of the inner nuclear layer (INL), and on OCTA, there was good perfusion of the superficial capillary plexus (SCP), but deficits were found in the perifoveal deep capillary plexus (DCP).
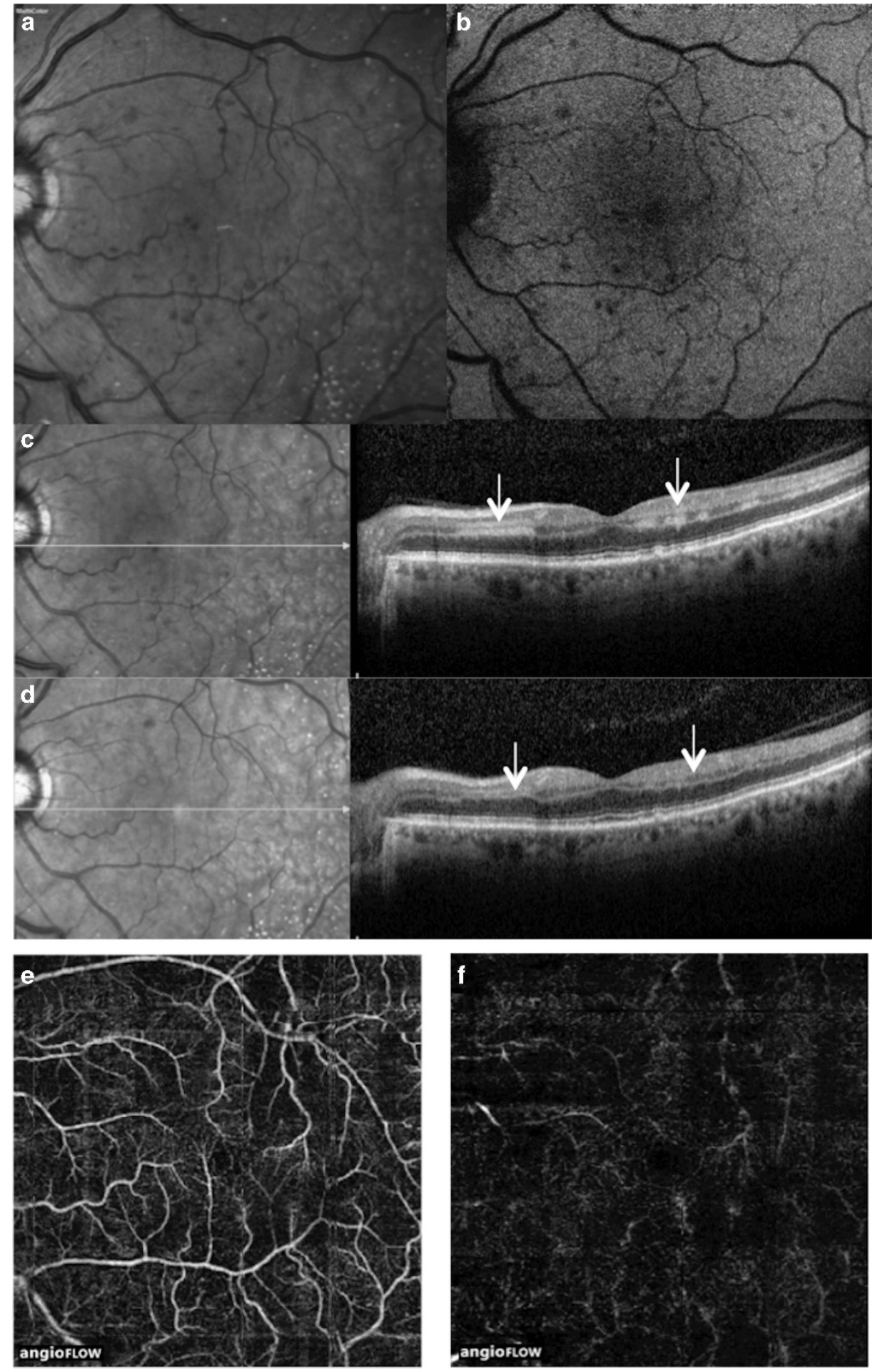

Figure 2 Case 2. Multimodal imaging of left eye. At presentation, BCVA was 83 ETDRS letters; CFP (a) revealed venous engorgement and widespread retinal hemorrhages visible also on blue fundus autofluorescence (b); SD-OCT scan (c) showed hyperreflective band-like lesions involving the middle layers of the retina at the level of the INL (arrows). After 2 months, BCVA was 77 letters; SD-OCT scan (d) revealed thinning of the INL (arrows); OCTA showed mild attenuation of the SCP (e) diffuse patchy areas of attenuation and pruning of the DCP (f). 

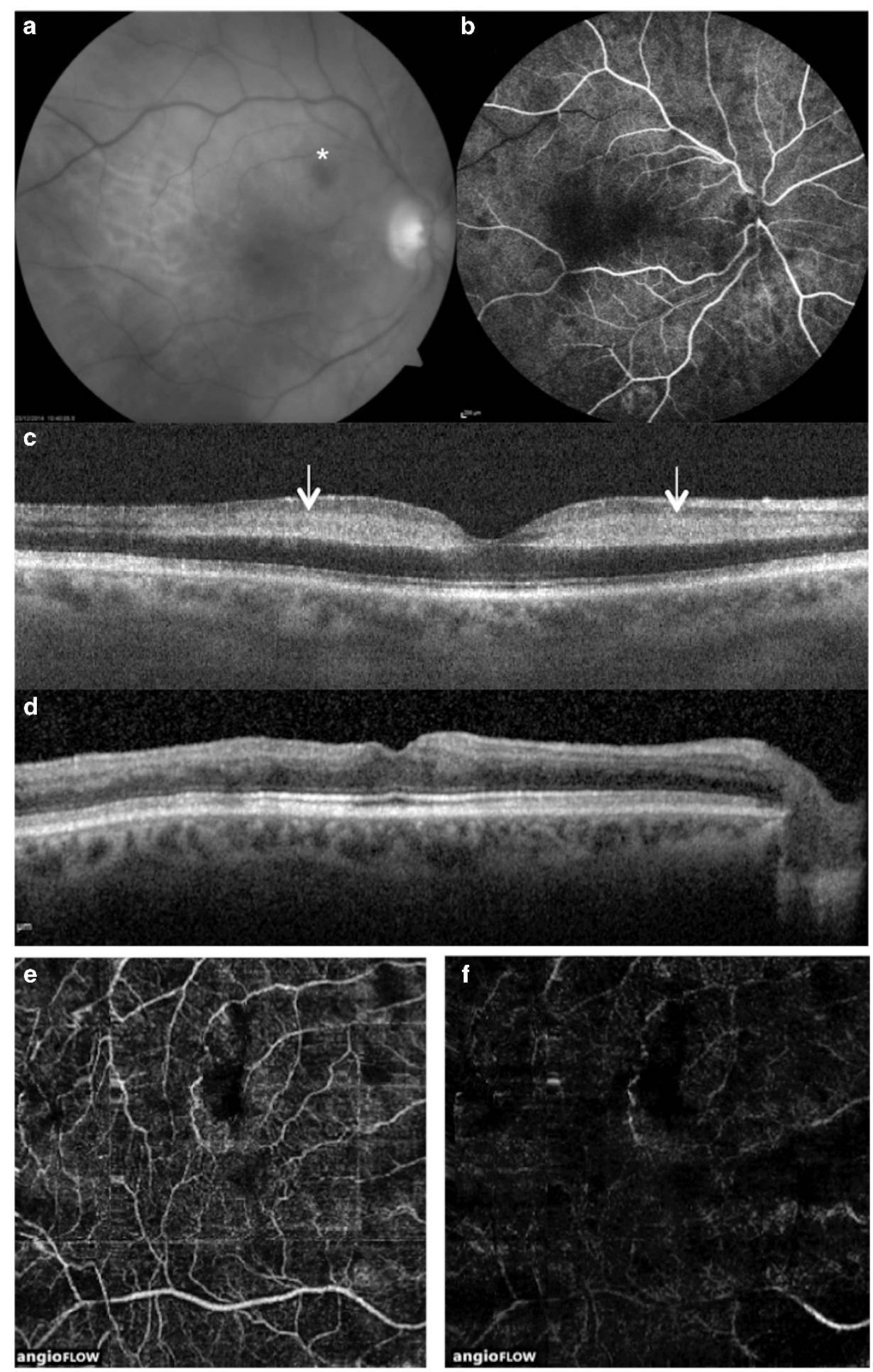

Figure 3 Case 3. Multimodal imaging of right eye. BCVA at presentation was 26 ETDRS letters; CFP (a) revealed venous engorgement and a retinal hemorrhage at the posterior pole $\left(^{*}\right)$; filling was slow during venous phases of FA (b), which revealed no areas of capillary closure; SD-OCT scan (c) showed hyperreflective band-like lesions involving the middle layers of the retina at the level of the INL (arrows). After 9 months, BCVA was eight ETDRS letters; SD-OCT scan (d) revealed marked thinning of the INL; OCTA showed mild attenuation of the SCP (e) and extensive capillary dropout of the DCP (f). 


\section{Case 2}

A 71-year-old man was referred for sudden acute-onset nonspecific visual loss in the left eye (LE) (Figure 2). Apart from controlled hypertension, his medical history was unremarkable. BCVA was 84 letters in the RE and 83 in the LE. Fundus examination of the LE revealed a CRVO. No abnormalities were detected in the RE. SD-OCT scans showed typical features of PAMM in the LE. After 2 months, thinning of INL was noted on SD-OCT scans. BCVA was stable in the RE and was 77 letters in the LE. On OCTA, there were patchy areas of attenuation and pruning of the DCP of the LE.

\section{Case 3}

A 72-year-old man was referred for a sudden onset drop of vision in RE (Figure 3). He suffered from controlled hypertension and he had a history of myocardial infarction. His ocular history was unremarkable. BCVA was 30 letters in RE and 82 letters in LE. Fundus examination of the RE revealed venous engorgement and a retinal hemorrhage at the posterior pole. Filling was slow during venous phases of fluorescein angioraphy (FA) and the SD-OCT scan showed typical features of PAMM. No abnormalities were detected in the fellow eye. After 9 months, SD-OCT showed thinning of INL and BCVA had decreased to eight letters in the RE and was stable in the LE. At this visit, extensive dropout of the DCP of the RE was seen on OCTA.

\section{Discussion}

The retinal vasculature is composed of superficial, intermediate, and deep capillary plexus. Isolated ischemia of the intermediate and DCP is thought to be responsible for the manifestations of PAMM. This hypothesis was based on the SD-OCT evidence of INL thinning that appears during the natural course of this disease. The same hypothesis has been further supported by a recent study showing that PAMM lesions may occur in angiographically silent retinal artery occlusion. ${ }^{6}$ FA is currently the gold standard to image retinal ischemia. This imaging modality allows visualization of the SCP in excellent detail, however, the intermediate and DCP are not seen. ${ }^{7}$ With increasing scan speeds, high resolution tomographic data acquisition and use of phase variance or decorrelation signals from blood cells within the vasculature and three-dimensional images of the retinal vessels can be generated. Essentially, en face visualization, layer by layer of the retina, with its constituent vascular beds is now a reality permitting better clinicopathological correlations to be made even in vivo. ${ }^{7}$
Recently, case reports and case series have imaged PAMM with OCTA-demonstrating areas of attenuation and pruning of the DCP. ${ }^{8-12}$

However, little is known about the natural course of PAMM secondary to CRVO and no correlation between the natural course of the visual acuity and the extension of the DCP has been reported so far. In our series, we showed that the visual outcome of PAMM associated with CRVO may vary, and in our cases, the extent of the DCP involvement on OCTA reflected the extent of the visual acuity impairment.

\section{Summary}

What was known before

- Paracentral acute middle maculopathy (PAMM) is a recently described optical coherence tomography (OCT) manifestation related to ischemia of the deep retinal circulation. Unlike fluorescein angiography, OCT angiography (OCTA) is able to visualize and delineate the extent of ischemia in the deep capillary plexus (DCP) in patients with PAMM.

What this study adds

- The natural course of visual acuity in patients with PAMM secondary to CRVO may vary. In these patients, the extent of the DCP involvement on OCTA may reflect the extent of the visual acuity impairment.

\section{Conflict of interest}

The authors declare no conflict of interest.

\section{Acknowledgements}

No author received financial or material support for the research and the work. No author has financial or proprietary interest related to the research.

\section{References}

1 Yu S, Wang F, Pang CE, Yannuzzi LA, Freund KB. Multimodal imaging findings in retinal deep capillary ischemia. Retina 2014; 34: 636-646.

2 Sarraf D, Rahimy E, Fawzi AA, Sohn E, Barbazetto I, Zacks DN et al. Paracentral acute middle maculopathy: a new variant of acute macular neuroretinopathy associated with retinal capillary ischemia. JAMA Ophthalmol 2013; 131: 1275-1287.

3 Rahimy E, Kuehlewein L, Sadda SR, Sarraf D. Paracentral acute middle maculopathy: what we knew then and what we know now. Retina 2015; 35: 1921-1930.

4 Rahimy E, Sarraf D, Dollin ML, Pitcher JD, Ho AC. Paracentral acute middle maculopathy in nonischemic central retinal vein occlusion. Am J Ophthalmol 2014; 158: 372-380. 
5 Chen X, Rahimy E, Sergott RC, Nunes RP, Souza EC, Choudhry $\mathrm{N}$ et al. Spectrum of retinal vascular diseases associated with paracentral acute middle maculopathy. Am J Ophthalmol 2015; 160: 26-34.

6 Yu S, Pang CE, Gong Y, Freund KB, Yannuzzi LA, Rahimy E et al. The spectrum of superficial and deep capillary ischemia in retinal artery occlusion. Am J Ophthalmol 2015; 159: 53-63.

7 Spaide RF, Klancnik JM, Cooney MJ. Retinal vascular layers imaged by fluorescein angiography and optical coherence tomography angiography. JAMA Ophthalmol 2015; 133: 45-50.

8 Christenbury JG, Klufas MA, Sauer TC, Sarraf D. OCT angiography of paracentral acute middle maculopathy associated with central retinal artery occlusion and deep capillary ischemia. Ophthalmic Surg Lasers Imaging Retina 2015; 46: 579-581.

9 Dansingani K, Inoue M, Engelbert M, Freund KB. Optical coherence tomographic angiography shows reduced deep capillary flow in paracentral acute middle maculopathy. Eye (Lond) 2015; 29: 1620-1624.

10 Khan MA, Rahimy E, Shahlaee A, Hsu J, Ho AC. En face optical coherence tomography imaging of deep capillary plexus abnormalities in paracentral acute middle maculopathy. Ophthalmic Surg Lasers Imaging Retina 2015; 46: 972-975.

11 Sridhar J, Shahlaee A, Rahimy E, Hong BK, Khan MA, Maguire JI et al. Optical coherence tomography angiography and en face optical coherence tomography features of paracentral acute middle maculopathy. Am J Ophthalmol 2015; 160: 1259-1268.

12 Nemiroff J, Kuhlewein L, Rahimy E, Tsui I, Doshi R, Gaudric A et al. Assessing deep retinal capillary ischemia in paracentral acute middle maculopathy by optical coherence tomography angiography. Am J Ophthalmol 2015; 162: 121-132.e1. 